\title{
Clinical commentary
}

Epileptic Disord 2017; 19 (4): 461-4

\section{Seizure semiology of anti-LGl1 antibody encephalitis}

\author{
Nicholas J. Beimer, Linda M. Selwa \\ Department of Neurology, University of Michigan, Michigan, USA
}

Received May 14, 2017; Accepted October 08, 2017

\begin{abstract}
Limbic encephalitis associated with anti-LGI1 antibody (LGI1 encephalitis) presents with a variety of features, the most prominent of which include seizures and progressive disturbance of memory and behaviour. Although varied in semiology, recognition of the pattern of seizures in LGl1 encephalitis is important, as early diagnosis and definitive treatment may prevent subsequent development of cognitive impairment. We present a patient with LGI1 encephalitis and "faciobrachial dystonic seizures-plus", which began as classic faciobrachial dystonic seizures and progressed to focal seizures with impaired awareness, dacrystic/gelasticlike outbursts, ictal speech, manual automatisms, and autonomic signs (tachycardia). Recognition of the broad range of seizure types associated with LGI1 encephalitis is crucial for early diagnosis and definitive treatment. [Published with video sequence on www.epilepticdisorders.com]
\end{abstract}

Key words: LGI1, encephalitis, faciobrachial, dystonic, ictal speech, temporal

Limbic encephalitis associated with leucine-rich glioma inactivated-1 (LGI1) antibody (LGI1 encephalitis) presents with a variety of features, including seizures and progressive disturbance of memory and behaviour. The most common seizure type associated with LGI1 antibody is the faciobrachial dystonic seizure (FBDS), although other seizure types can develop with progression of limbic encephalitis (Irani et al., 2011; Aurangzeb et al., 2017). The seizures of patients with LGl1 encephalitis occur frequently (10100 per day), are poorly responsive to antiseizure medications, and are sensitive to immunotherapy, such as steroids, IVIg, plasmapheresis, and rituximab (Shin et al., 2013; Irani et al., 2014). Recognition of the pattern of seizures in LGI1 encephalitis is important, as early diagnosis and definitive treatment may prevent subsequent development of cognitive impairment (Irani et al., 2013; Finke et al., 2017).

\section{Case study}

The patient was a 51-year-old woman with a history of asthma and hypothyroidism, who presented with an episodic tingling sensation in her arms and hands that progressed to stereotyped hand wringing movements and playing with her hair over a period of three months. During these events, she could have repetitive mouth movements, made "weird" facial expressions, and developed yelling 
verbal outbursts, of which she had no recollection. The verbal outbursts could also occur during sleep, causing frequent nocturnal arousals. The patient additionally reported confusion and memory impairment which significantly affected her work performance.

Initial workup included a routine EEG and MRI of the brain. The EEG was significant for occasional left temporal intermittent rhythmic delta activity (TIRDA) and two focal subclinical seizures, with retained awareness arising independently from the left and right temporal regions. The patient correctly recalled a memory phrase given during both seizures. The MRI was significant only for a partially empty sella and a benignappearing lesion in the left sphenoid sinus. There was no abnormal contrast enhancement or structural abnormality noted. Levetiracetam was started at $1,500 \mathrm{mg} /$ day, although events continued to occur daily. An autoimmune encephalitis panel subsequently resulted positive for LGl1 antibody.

The patient was admitted for diagnostic long-term EEG monitoring and treatment with high-dose IV methylprednisolone. During the first two days of monitoring, 24 seizures were recorded during both wakefulness and sleep. There were two distinct seizure types with onset independently occurring over the right and left temporal regions. Right temporal-onset seizures included right-hand manual automatisms, an unusual facial expression or dystonic facial contraction, tachycardia, and outbursts of non-sensical ictal speech. Electrographically, the onset of these seizures occurred broadly over the right temporal derivations as rhythmic delta activity, occasionally evolving to the left temporal lobe. Left temporal-onset seizures were associated with non-fluent aphasia and were occasionally preceded by nose rubbing with the left hand. Electrographically, these seizures were characterized by rhythmic delta activity broadly over the left temporal derivations, with a significant electrographic delay from clinical onset. Most of the recorded seizures were right temporal-onset with occasional evolution to the left temporal region. A minority of seizures appeared to only have an electrographic correlate over the left temporal lobe. All nocturnal seizures occurred during non-rapid eye movement (NREM) sleep. The interictal EEG also demonstrated frequent independent left and right intermittent bitemporal, bifrontal, and biparasagittal semi-rhythmic delta activity.

On the first day of admission, the patient was started on IV methylprednisolone at $1 \mathrm{~g}$ daily for five days. No malignancy was seen on CT of the chest. On the second day, lacosamide, $100 \mathrm{mg}$ twice daily, was started. On hospital rounds, the patient was agitated, delusional, and had removed her own EEG leads. She believed a physician told her that she would be discharged and had already packed her belongings and called for a ride home. EEG monitoring was restarted and there were no further seizures or interictal slowing recorded up to Day 5. Levetiracetam was discontinued due to concern for mood and behavioural side effects. The patient was discharged on lacosamide $(200 \mathrm{mg}$, twice daily) and oral prednisone (60 $\mathrm{mg}$, daily), and during follow-up, one week after discharge, the patient remained seizure-free and reported an improvement in her memory, although still had difficulty remembering details of the past month. Neuropsychological evaluation was organised and a long-term tapering schedule for prednisone was planned, in which prednisone was reduced by $10 \mathrm{mg} /$ day each month.

\section{Discussion}

FBDS are pathognomonic for limbic encephalitis associated with LGl1 antibodies (Sen et al., 2014). Despite this association, other seizure types have been described, including those with impaired awareness and autonomic, motor, or gelastic features (van Sonderen et al., 2016; Aurangzeb et al., 2017). Recognition of FBDS, along with the spectrum of seizure types associated with LGl1 antibodies, may aid in early diagnosis and definitive treatment.

Our case demonstrates that LGI1 encephalitis may present with a broad range of seizure types. The accompanying video shows several examples of FBDS that progress to focal seizures with impaired awareness, dacrystic/gelastic-like outbursts, ictal speech, manual automatisms, and autonomic changes (tachycardia). We propose naming these episodes "faciobrachial dystonic seizures-plus" (FBDS-plus), as the semiology progresses to include features beyond those of typical FBDS. Furthermore, although ictal speech arrest has been described, to our knowledge, ictal speech has not been previously reported as a feature of seizures associated with LGl1 encephalitis (Irani et al., 2013).

There are several features that made diagnosis challenging in this patient. Although there was an ictal EEG correlate during this patient's seizures, it was poorly formed and/or significantly delayed after clinical onset, and there were also no interictal or ictal epileptiform discharges despite frequent seizures having been seen in the first 24 hours of recording. Despite the subtle ictal correlate, we believe the EEG changes met the criteria for seizures as the tracing demonstrated progression in morphology and location of rhythmic delta activity, independently over the right and left temporal regions. Rapid eye movement sleep behaviour disorder (RBD) should also be considered in the differential diagnosis for this patient's nocturnal events, as it has been described in patients with voltage-gated potassium channel antibody-associated limbic encephalitis (Iranzo et al., 2006). However, the nocturnal arousals in 
this patient did not occur during REM and were associated with abnormal EEG changes, which excludes RBD. Our patient also did not have any MRI abnormalities associated with LGI1 encephalitis and FBDS, such as medial temporal lobe T2 hyperintensity or basal ganglia T1 hyperintensity (Varley and Irani, 2015; Flanagan et al., 2015). Nevertheless, the pattern of frequent seizures, absence of a well-defined ictal EEG correlate, and poor response to antiseizure medications should prompt consideration and further workup for an autoimmune aetiology. Because seizures associated with LGI1 antibody may precede the onset of cognitive symptoms, and early diagnosis and definitive treatment may prevent subsequent development of cognitive impairment, recognition of the electroclinical syndrome associated with LGI1 antibodies is important and warrants further investigation (Irani et al., 2011; Irani et al., 2013; Finke et al., 2017).

\section{Legend for video sequence}

Digital EEG recording with time-locked video of seizures in a patient with LGI1 encephalitis. Electrodes were placed in accordance with the 10-20 international system of electrode placement and included 21 channels, single-lead EKG monitoring, as well as temporal electrodes (T1 and T2). A total of four seizures are shown. The first seizure occurs out of NREM sleep with semiology of right-hand and oral automatisms, variably comprehensible ictal speech, vocalization, and distressed affect, with an ictal EEG correlate of rhythmic delta activity over the right hemisphere, maximal in the right temporal region. The second seizure occurs during wakefulness and is characterized by the patient starting to ask a question when she develops a brief dystonic contraction of the left side of the face, followed by non-fluent aphasia with an ictal EEG correlate of rhythmic delta activity over the left temporal region. The third seizure occurs during wakefulness and starts with rapid right-hand automatisms, followed by a grimacing facial expression during conversation, with an ictal EEG correlate of rhythmic right temporal delta activity. The fourth seizure starts with right-hand automatisms, followed by ictal speech, distressed affect, and yelling, with an ictal EEG correlate starting as rhythmic delta activity over the right temporal region, evolving to the left temporal region.

Key words for video research on www.epilepticdisorders.com

Phenomenology: face, distonia

Localisation: temporal lobe (bilateral)

Syndrome: focal non-idiopathic temporal

Aetiology: encephalitis
In summary, we highlight a patient with LGI1 encephalitis and FBDS-plus, which began as classic FBDS and progressed to focal seizures with impaired awareness and included features of dacrystic/gelasticlike outbursts, ictal speech, manual automatisms, and autonomic changes. Recognition of the broad range of seizure types associated with LGI1 encephalitis is crucial for early diagnosis and definitive treatment.

\section{Supplementary data.}

Summary didactic slides are available on the www.epilepticdisorders.com website.

\section{Acknowledgements and disclosures.}

The authors would like to thank the faculty and staff of the EEG laboratory and epilepsy monitoring unit at Michigan Medicine for their dedication in providing excellent patient care, education, and research.

None of the authors have any conflict of interest to declare.

\section{References}

Aurangzeb S, Symmonds M, Knight RK, et al. LGl1-antibody encephalitis is characterised by frequent, multifocal clinical and subclinical seizures. Seizure 2017; 50:14-7.

Flanagan EP, Kotsenas AL, Britton JW, et al. Basal ganglia T1 hyperintensity in LGl1-autoantibody faciobrachial dystonic seizures. Neurol Neuroimmunol Neuroinflamm 2015; 2: e161.

Finke C, Prüss $\mathrm{H}$, Heine J, et al. Evaluation of cognitive deficits and structural hippocampal damage in encephalitis with leucine-rich, glioma-inactivated 1 antibodies. JAMA Neurol 2017; 74:50-9.

Irani SR, Michell AW, Lang B, et al. Faciobrachial dystonic seizures precede LGI1 antibody limbic encephalitis. Ann Neurol 2011; 69: 892-900.

Irani SR, Stagg CJ, Schott JM, et al. Faciobrachial dystonic seizures: the influence of immunotherapy on seizure control and prevention of cognitive impairment in a broadening phenotype. Brain 2013; 136: 3151-62.

Irani SR, Gelfand JM, Bettcher BM, et al. Effect of rituximab in patients with leucine-rich, glioma-inactivated 1 antibody-associated encephalopathy. JAMA Neurol 2014; 71: 896-900.

Iranzo A, Graus F, Clover L, et al. Rapid eye movement sleep behavior disorder and potassium channel antibodyassociated limbic encephalitis. Ann Neurol 2006; 59: 178-81.

Sen A, Wang J, Laue-Gizzi H, et al. Pathognomonic seizures in limbic encephalitis associated with anti-LGl1 antibodies. Lancet 2014; 383: 2018.

Shin YW, Lee ST, Shin JW, et al. VGKC-complex/LGI1antibody encephalitis: clinical manifestations and response to immunotherapy. J Neuroimmunol 2013; 265: 75-81. 
van Sonderen A, Thijs RD, Coenders EC, et al. Anti-LGl1 encephalitis: clinical syndrome and long-term follow-up. Neurology 2016; 87: 1449-56.
Varley JA, Irani SR. Treating seizures and preventing amnesia in LGI1-antibody encephalitis: a new MRI signature? Neurol Neuroimmunol Neuroinflamm 2015; 2: e182.

\section{TEST YOURSELF}

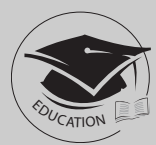

(1) Antibodies associated with faciobrachial dystonic seizures include which of the following?

A. Type 1 anti-neuronal nuclear (ANNA-1 or anti-Hu)

B. Leucine-rich glioma-inactivated protein 1 (LGI)

C. P/Q-type voltage-gated calcium channel (VGCC)

D. N-methyl-D-aspartate receptor (NMDAR)

E. Thyroperoxidase (TPO)

(2) Which of the following is a typical frequency of faciobrachial dystonic seizures associated with anti-LGI1 antibody encephalitis?

A. 1 seizure per week

B. 1 seizure per day

C. 1-10 seizures per day

D. 10-100 seizures per day

E. $100-200$ seizures per day

(3) Which of the following is the most effective form of treatment for seizures associated with anti-LGI1 antibody encephalitis?

A. Carbamazepine

B. Levetiracetam

C. Immunotherapy (i.e. steroids, IVIg, plasmapheresis)

D. Resective epilepsy surgery

E. Ketogenic diet

Note: Reading the manuscript provides an answer to all questions. Correct answers may be accessed on the website, www.epilepticdisorders.com, under the section "The EpiCentre". 